\title{
NUMERICALLY DETERMINING SOLUTIONS OF SYSTEMS OF POLYNOMIAL EQUATIONS
}

\author{
T. Y. LI, TIM SAUER AND JAMES A. YORKE
}

In this report we suggest some efficient algorithms for numerically determining all solutions of a system of $n$ polynomial equations in $n$ unknowns. Such systems are common in many fields of engineering. When all equations are linear, there is at most one isolated solution. In the general case, even the number of solutions can be difficult to predict.

By a classical theorem of Bézout, the number of isolated solutions of the system is bounded above by the total degree $d=d_{1} \cdots d_{n}$, where $d_{i}$ denotes the degree of the $i$ th equation. Empirically, we find that most systems arising in applications have fewer than $d$ solutions. We call such systems deficient. Our purpose is to describe some methods for which the computational work, instead of being proportional to the total degree, is proportional to the actual number of solutions.

The first practical computer-implementable method for numerically solving polynomial systems was introduced in [D] (see also [GZ]) using arguments based on algebraic geometry. The authors of [CMY] reformulated this result, replacing the algebraic geometry arguments with a general version of Sard's Theorem. The article [AG] presents a survey of homotopy methods for numerically solving systems of equations.

Elimination theory is the classical approach to solving systems of polynomial equations, but its reliance on symbolic manipulation makes it seem unsuitable for all but small problems. Moreover, the method (unlike Gaussian elimination for the linear case) reduces the problem to the ill-conditioned problem of numerically solving a high-degree polynomial equation in one variable. In this paper, we use elimination theory and other techniques from algebraic geometry as theoretical tools, but our algorithms avoid the computing of resultants.

1. Random product homotopy. Let

$$
\begin{gathered}
p_{1}\left(x_{1}, \ldots, x_{n}\right)=0, \\
\vdots \\
p_{n}\left(x_{1}, \ldots, x_{n}\right)=0
\end{gathered}
$$

be the system of polynomial equations to be solved, i.e., $P: \mathbf{C}^{n} \rightarrow \mathbf{C}^{n}, P=$ $\left(p_{1}, \ldots, p_{n}\right)$. Although we are only interested in computing the solutions in

Received by the editors December 20, 1986.

1980 Mathematics Subject Classification (1985 Revision). Primary 65H10, 90B99, 65H15.

The authors were supported in part by a contract with the Applied and Computational Mathematics Program of DARPA. 
$\mathbf{C}^{n}$, it is useful to view the system $P(x)=0$ in projective space $\mathbf{C P}^{n}$. Let $V(P)_{\infty}$ denote the solutions at infinity. Define the random product system

$$
\begin{gathered}
q_{1}\left(x_{1}, \ldots, x_{n}\right)=\left(L_{11}(x)+b_{11}\right) \cdots\left(L_{1 d_{1}}(x)+b_{1 d_{1}}\right) \\
\vdots \\
q_{n}\left(x_{1}, \ldots, x_{n}\right)=\left(L_{n 1}(x)+b_{n 1}\right) \cdots\left(L_{n d_{n}}(x)+b_{n d_{n}}\right)
\end{gathered}
$$

where the $L_{i j}(x)$ are fixed nonzero linear forms, and $b_{i j} \in \mathbf{C}$. Note that $Q(x)=0$ is trivial to solve. The choice of the $L_{i j}$ will be tailored to the problem $P(x)=0$ to be solved, and the number of solutions of $Q(x)=0$ will depend on the $L_{i j}$. Let $H: \mathbf{C}^{n} \times[0,1] \rightarrow \mathbf{C}^{n}$ be defined by $H(x, t)=$ $(1-t) a Q(x)+t P(x)$.

The object is to prove the existence of smooth paths connecting the trivial solutions of $Q(x)=0$ to the unknown solutions of $P(x)=0$. More precisely, we want to prove that the following two properties hold.

1. Smoothness property. The solutions of $H(x, t)=0$ with $0 \leq t<1$ consist of a finite number of smooth paths, each parametrized by $t$ in $[0,1)$.

2. Accessibility property. Every isolated solution of $H(1, x) \equiv P(x)=0$ is reached by some path originating at $t=0$. It follows that this path starts at a solution of $H(0, x) \equiv Q(x)=0$.

RANDOM PRODUCT THEOREM. (a) For any choice of the $L_{i j}(x)$, there exists an open dense full-measure subset $U$ of $\mathbf{C}^{d+1}$ such that for $\left(a, b_{i j}\right) \in U$, the smoothness property holds.

(b) Assume that the $L_{i j}$ are chosen so that for an open dense subset of $\left(b_{i j}\right), V(Q)_{\infty} \subseteq V(P)_{\infty}\left(\right.$ as sets) and $V(Q)_{\infty}$ is a smooth submanifold of $\mathbf{C P}^{n}$. Then there exists an open dense full-measure subset $U$ of $\mathbf{C}^{d+1}$ such that for $\left(a, b_{i j}\right) \in U$, the accessibility property holds.

In practice, the complex numbers $a, b_{i j}$ are chosen at random. Once the initial points at $t=0$ are known, the continuation paths can be followed from $t=0$ to $t=1$ using standard numerical techniques involving differential equation solvers [AG]. The beginning points of the paths are the solutions of $Q(x)=0$, which are easily found because of the product structure of $Q$.

The following example illustrates a homotopy in the spirit of $[\mathbf{D}, \mathbf{C M Y}$, GZ, L].

EXAMPLE 1. The above theorem can be used to find all solutions of any polynomial system $P(x)=0$ by setting $L_{i j}(x)=x_{i}$ for $i=1, \ldots, n$, and $j=1, \ldots, d_{i}$. Then $V(Q)_{\infty}=\varnothing$. The hypotheses of the theorem are vacuously satisfied. The system

$$
\begin{gathered}
q_{1}(x)=\prod_{j=1}^{d_{1}}\left(x_{1}+b_{1 j}\right) \\
\vdots \\
q_{n}(x)=\prod_{j=1}^{d_{n}}\left(x_{n}+b_{n j}\right)
\end{gathered}
$$

has $d=d_{1} \cdots d_{n}$ solutions, which originate $d$ paths leading to all isolated solutions of $P(x)=0$. In this example, the amount of computation required 
to find all solutions is roughly proportional to $d$. If the system is deficient, the paths which do not converge to a solution in $\mathbf{C}^{n}$ diverge to infinity, representing wasted computation.

EXAMPLE 2. For a typical example of a deficient system, suppose that each of the highest-degree monomials of each $p_{i}(x)$ contains at least one of the variables of the distinguished subset $\left\{x_{1}, \ldots, x_{m}\right\}$, where $m<n$. The deficiency of this system is due to the existence of a manifold of solutions at infinity of dimension at least $n-m-1$. A Chern class formula from intersection theory $[\mathbf{F}]$ shows that the contribution of this set to the Bézout number of the system is at least $s$, where $s$ is the coefficient of $t^{n-m-1}$ in the Maclaurin series expansion of

$$
(1+t)^{-m-1} \prod_{i=1}^{n}\left(1+d_{i} t\right)
$$

Thus the number of solutions in $\mathbf{C}^{n}$ of $P(x)=0$ is at most $N=d-s$, less than the total degree. Given a system of this type, define $Q(x)$ by

$$
q_{i}(x)=\left(\sum_{j=1}^{m} c_{i j 1} x_{j}+b_{i 1}\right) \prod_{k=2}^{d_{i}}\left(\sum_{j=1}^{n} c_{i j k} x_{j}+b_{i k}\right),
$$

where the $c_{i j k}$ and $b_{i j}$ are chosen randomly. Then the theorem shows that this homotopy provides paths that reach all isolated solutions of $P(x)=0$. One can check that $Q(x)=0$ has exactly $N$ solutions, and therefore the theorem produces $N$ paths. In the process of reproving that there are at most $N$ isolated solutions, we get a computer-implementable method for finding the solutions.

For example, let $P(x)$ be the system

$$
\begin{gathered}
x_{1}\left(\alpha_{11} x_{1}+\cdots+\alpha_{1 n} x_{n}\right)+L_{1}\left(x_{1}, \ldots, x_{n}\right)+\beta_{1}=0, \\
\vdots \\
x_{1}\left(\alpha_{n 1} x_{1}+\cdots+\alpha_{n n} x_{n}\right)+L_{n}\left(x_{1}, \ldots, x_{n}\right)+\beta_{n}=0,
\end{gathered}
$$

where $L_{i}(x)$ are arbitrary linear forms, $\beta_{i} \in \mathrm{C}$. The total degree is $d=2^{n}$; however, $P(x)=0$ has as most $n+1$ solutions. The theorem provides $n+1$ paths leading to all solutions of $P(x)=0$. For large $n$, this is the difference between a problem which is tractable and one which is not.

2. Cheater's homotopy. In some cases the structure of $P(x)$ is difficult to analyze, and as a result a good choice of the random product in the above theorem may be difficult to find, or may not even exist. We give an alternative approach for such cases which is especially useful when deficient systems of a fixed general form must be solved repetitively with varying coefficients.

Suppose

$$
\begin{gathered}
p_{1}\left(c_{1}, \ldots, c_{M}, x_{1}, \ldots, x_{n}\right)=0, \\
\vdots \\
p_{n}\left(c_{1}, \ldots, c_{M}, x_{1}, \ldots, x_{n}\right)=0
\end{gathered}
$$

is a system of polynomial equations, where we consider the $c_{i}$ as coefficients and the $x_{i}$ as variables. The idea is to use the method of Example 1 to solve 
the system with random coefficients $c_{i}^{*}$ the first time, following all $d$ paths, and then for subsequent runs, with various values of $c_{i}$, to use these (perhaps fewer than $d$ ) solutions as "seeds" to initialize paths. If the structure of the system causes it to be deficient, this method results in fewer paths which need to be followed in subsequent runs. The precise formulation is as follows.

CHEATER'S HOMOTOPY THEOREM. There exists an open dense fullmeasure subset $U$ of $\mathbf{C}^{M+n}$ such tht for $\left(b_{1}^{*}, \ldots, b_{n}^{*}, c_{1}^{*}, \ldots, c_{M}^{*}\right) \in U$, the following holds:

(a) The set $X^{*}$ of solutions $x=\left(x_{1}, \ldots, x_{n}\right)$ of

$$
\begin{gathered}
q_{1}\left(x_{1}, \ldots, x_{n}\right)=p_{1}\left(c_{1}^{*}, \ldots, c_{M}^{*}, x_{1}, \ldots, x_{n}\right)+b_{1}^{*}=0, \\
\vdots \\
q_{n}\left(x_{1}, \ldots, x_{n}\right)=p_{n}\left(c_{1}^{*}, \ldots, c_{M}^{*}, x_{1}, \ldots, x_{n}\right)+b_{n}^{*}=0
\end{gathered}
$$

consists of a finite number of points (at most $d$ ).

(b) The smoothness and accessibility properties hold for the homotopy

$$
H(x, t)=P\left((1-t) c_{1}^{*}+t c_{1}, \ldots,(1-t) c_{M}^{*}+t c_{M}, x_{1}, \ldots, x_{n}\right)+(1-t) b^{*} \text {, }
$$

where $b^{*}=\left(b_{1}^{*}, \ldots, b_{n}^{*}\right)$. It follows that every solution of $P(x)=0$ is reached by a path beginning at a point of $X^{*}$.

NOTE. The theorem requires the $c_{i}^{*}$ to be complex numbers, even if the specific coefficients $c_{i}$ in the problem to be solved are real.

EXAMPLE 3. A useful application of this theorem is to the robot arm equations of [MT]. This highly deficient system consists of eight fairly complicated quadratic polynomials in eight unknowns. Although the total degree is $2^{8}=256$, the system has at most 32 solutions. In fact, the set $X^{*}$ of the theorem consists of 32 points. For each new set of coefficients for which the system needs to be solved (corresponding, for example, to a movement of the hand from one point to another), only 32 new paths need to be followed to find all solutions.

3. Methods of proof. Part (a) of the Random Product Theorem is a matter of the implicit function theorem. It suffices to verify that the rank of the Jacobian $\partial H / \partial x$ is equal to $n$ at each finite solution $(x, t)$. For $t=0$, one uses Bertini's theorem successively on $q_{1}, \ldots, q_{n}$. The base locus lies at infinity because of the structure of $Q$. For general $t$, the existence of resultant polynomials guarantees the rank is $n$ for vectors $\left(b_{i j}\right)$ chosen from an open dense full-measure subset of $\mathbf{C}^{d}$. The proof of the smoothness property for the Cheater's Homotopy Theroem is similar.

Now that the solution paths are smooth one-manifolds, it suffices to show that the solution paths emanating from each solution of $P(x)=0$ (at $t=1$ ) stay finite for $t \in[0,1)$ and reach a (finite) solution of $Q(x)=0$ (at $t=0$ ). Let $\left\langle f_{1}, \ldots, f_{k}\right\rangle$ denote the ideal generated by the homogeneous polynomials $f_{1}, \ldots, f_{k}$ and let $\left\langle f_{1}, \ldots, f_{k}\right\rangle_{m}$ denote the degree $m$ part. Let $\tilde{h}$ denote the homogenization (by $x_{0}$ ) of the polynomial $h$. From the hypotheses of our theorems, one shows that for a generic choice of constants, $\operatorname{dim}_{\mathbf{C}}\left\langle\tilde{h}_{1}, \ldots, \tilde{h}_{n}, x_{0}^{K}\right\rangle_{m}$ is constant for $t \in[0,1)$, for sufficiently large $K, m$. Then the theorems follow from this key lemma: 
LEMMA. Let $H: \mathbf{C}^{n} \times U \rightarrow \mathbf{C}^{n}$, where $U$ is an open subset of $\mathbf{R}, H$ is continuous in $t$, and $H(x, t)$ is a polynomial map for each $t$. Suppose there are integers $K<m$ such that $\operatorname{dim}_{\mathbf{C}}\left\langle\tilde{h}_{1}, \ldots, \tilde{h}_{n}, x_{0}^{k}\right\rangle_{m}$ is independent of $t \in U$ and $k \in\{K, K+1\}$. Then no path of solutions of $H(x, t)=0$ parametrized by $t$ tends to infinity as $t \rightarrow t_{0} \in U$.

\section{REFERENCES}

[AG] E. Allgower and K. Georg, Simplicial and continuation methods for approximating fixed points and solutions to systems of equations, SIAM Review 22 (1980), 28-85.

[CMY] S. N. Chow, J. Mallet-Paret and J. Yorke, A homotopy method for locating all zeroes of a system of polynomials, Functional Differential Equations and Approximation of Fixed Points (H. O. Peitgen and H. O. Walter, eds.), Lecture Notes in Math., vol. 730, Springer-Verlag, Berlin and New York, 1979, pp. 77-88.

[D] F. Drexler, Eine Methode zur Berechnung Samlischer Losungen von Polynomgleichungssystemen, Numer. Math. 29 (1977), 45-58.

[GZ] C. B. Garcia and W. I. Zangwill, Finding all solutions to polynomial systems and other systems of equations, Math. Programming 16 (1979), 159-176.

[L] T. Y. Li, On Chow, Mallet-Paret and Yorke homotopy for solving systems of polynomials, Bull. Inst. Math. Acad. Sinica 11 (1983), 433-473.

[MT] A. P. Morgan and L.-W. Tsai, Solving the kinematics of the most general six- and five-degree-of-freedom manipulators by continuation methods, ASME J. of Mechanisms, Transmissions and Automation in Design 107 (1985), 48-57.

Department of Mathematics, Michigan State University, East lanSING, MICHIGAN 48824

Department of Mathematics, George Mason University, Fairfax, VirGINIA 22030

Institute For Physical SCIENCES AND TECHNOLOGY AND DEPARTMENT OF MAThematics, UNIVERSity OF MARYLAND, College PARK, MARYLAND 20742 
